Published online 2017 April 13.

\title{
Ultrasound-Guided Fine-Needle Aspiration of Thyroid Nodules: Relation Between Radiology Thyrads Score and Bethesda Pathology Score System
}

\author{
Reza Nafisi Moghadam, ${ }^{1}$ Mohammad Ghadrian,, ${ }^{2,}$ Seyadkazem Aghili, ${ }^{2}$ Mohammad \\ Sobhanardekani, ${ }^{2}$ Mohammadhosein Ahraryazdi, ${ }^{2}$ and Kazem Razavi $^{2}$ \\ ${ }^{1}$ Yazd University of Medical Sciences, Yazd, Iran \\ ${ }^{2}$ Resident of Radiology \\ "Corresponding author: Mohammad Ghadrian, Resident of Radiology. E-mail: Ghadrmed@gmail.com
}

Received 2016 December 21; Accepted 2017 February 08.

\begin{abstract}
Background: Although fine-needle aspiration biopsy (FNAB) is considered the standard for preoperative evaluation of thyroid nodules, the value of this has been questioned for different thyroid nodules size. Different pathology and radiological scoring may improve the accuracy of preoperative diagnosis of thyroid nudoles. The aim of this study was to evaluate the relation between radiology Thyrads score and Bethesda pathology score system size in two group size ( $\leq 10 \mathrm{~mm}$ and $>10 \mathrm{~mm}$ ) of thyroid nodule in US-FNAB.

Methods: There were 183 thyroid nodules between $3 \mathrm{~mm}$ to $35 \mathrm{~mm}$ who underwent US-FNAB from January 2015 to November 2016 in Shaheed Sadoughi hospital- Yazd. They were divided in to two groups (group A, 3-10 mm; group B, 11- 35 mm). The patients were studied according radiology Thyrads score and Bethesda pathology score system. The relation between radiology Thyrads score and Bethesda pathology score system according groups size was analyzed by SPSS21.

Results: The mean age ( \pm standard deviation) of patients was 44.5 ( \pm 12.8 years). Most of them were female 138 (83.6\%). The frequency of final pathology diagnoses were; 35(21.2\%) non diagnostic, 107 (64.8\%) benign, 13 (7.9\%) malignant and 10 (6.1\%) suspicious. no statically significance relation between thyrads score and nodule characteristics (solid/cyst (p-value: 0.555), calcification (P value: 0.558), lymph-adenopathy (P value: 0.521), ecogenicity (P value: 0.236$)$, margin (P value:0.149)). The spearman correlation between radiology thyroids score and Bethesda pathology score system was statistically significant. (Correlation coefficient: 0.202 , P value: $0.004)$.

Conclusions: There was a statistically significance correlation between Thyrads score and pathologic Bethesda scoring system. Decision making basis on the radiologic Thyrads score can improve the radiological diagnosis precision in nodular goiter patients.
\end{abstract}

Keywords: US-Guided Fine-Needle Aspiration Biopsy, Thyroid Nodules, Cytopathology, Bethesda Score System, Thyrads Score

This is an abstract presented in the 33rd Iranian congress of radiology (ICR) and the 15th congress of Iranian radiographic science association (IRSA) 